\title{
DIRECTORIES OF IOWA NEWSPAPERS, 1850-1869
}

\section{By Douglas C. McMurtrie}

The one completely dependable source of newspaper history is the files of the paper themselves. It was this source on which David C. Mott primarily depended in the preparation of his "Early Iowa Newspapers" which appeared in the Annals of Iowa of January, 1928. Lacking files or copies of the papers, however, the historian is forced to fall back on two secondary sources: (1) the county histories and (2) newspaper directories.

In 1869 began the annual series of Rowell's American Nezspaper Directory, which is invaluable to the student of newspaper history in the seventies and eighties. Prior to 1869, however, directories of newspapers were few and far between and, for some reason or other, they are exceedingly rare. It may even be that no copy of any one of the directories for the years between 1850 and 1869 is to be found in the state of Iowa.

These directories provide, however, exceedingly valuable material to the historian. In view of their rarity, therefore, I am here presenting the section relating to Iowa newspapers in four of them.

The listings are undoubtedly far from complete, for the compilers had no authoritative sources on which to draw, and correspondence was slow and difficult. The chief defects, however, will be found in omissions, the compilers apparently having sound evidence that most of the papers listed were being published. 'The Kennedy list, enumerating the newspapers published in 1850 , was based on the returns of the U.S. Census of 1850 ; the others were private compilations.

Obvious abbreviations have been spelled out in the present transcript. The county name is mostly enclosed in parenthesis. The title of the newspaper is here set in italics. We have followed their spelling of proper names and general style.

Kenneny, J. C. G., compiler. Catalogue of the Newspapers and

Periodicals published in the United States. . . Compiled 
from The United States Census Statistics of 1850, expressly for Livingston's Law Register for 1852. New York: John Livingston, 1852. (The Iowa section is on page 12.)

'Tipton (Cedar). T'ipton T'imes. Monthly. Miscellaneous in character. Circulation 400 .

Burlington (Des Moines). Burlington Harok Eye. Weekly. Whig. Circulation 1,200 .

Burlington (Des Moines). State Gazette. Weekly. Democrat. Circulation 900 .

Burlington (Des Moines). Telegraph. Tri-weekly. Neutral. Circulation 1,200 .

Burlington (Des Moines). Telegraph. Weekly. Neutral. Circulation 600.

Du Buque (Du Buque), Miners' Express. Weekly. Democrat. Circulation 1,120 .

Du Buque (Du Buque). Du Buque Tribune. Weekly. Whig. Circulation 1,104.

Du Buque (Du Buque). Du Buque Telegraph. Weekly. Whig. Circulation 700 .

Du Buque (Du Buque). Nordwestliche Democrat. Weekly. Democrat. Circulation -

M.t. Pleasant (Henry). Iowe Observer. Weekly. Whig. Circulation 500.

Mt. Pleasant (Henry). True Democrat. Weekly. Free Soil. Circulation 800 .

(Henry County). Western Evangelist. Monthly. Christian. Circulation 650.

Andrew (Jackson). Western Democrat. Weekly. Democrat. Circulation 500.

Fairfield (Jefferson). Iowa Sentinel. Weekly. Democrat. Circulation 550.

Fairfield (Jefferson), Fairfield Ledger. Weekly. Whig. Circulation

(Johnson County). Iowa Republican. Weekly. Whig. Circulation 1,040.

(Johnson County). Iowa Capital Reporter. Weekly. Democrat. Circulation 850 .

(Lee County) [Fort Madison]. Iorva Statesman. Weekly. Circulation 800 .

(Lee County) [Keokuk]. Whig and Register. Weekly. Whig. Circulation 1,600 .

(Lee County). Keokuk Dispatch. Weekly. Democrat. Circulation 1,000.

Wapello (Louisa). Louisa County Times. Weekly. Independent. Circulation - -

Oskaloosa (Mahaska); Oskaloosa Herald. Weekly. Whig. Circulation 500.

Muscatine (Muscatine). Democratic Inquirer. Weekly. Democrat. Circulation 500 . 
Muscatine (Muscatine). Muscatine Journal. Weekly. Circulation 300. Des Moines (Polk). Iowa Star. Weekly. Democrat. Circulation 510. (Polk County). Fort Des Moines Gazette. Weekly. Whig. Circulation 350.

Kanesville (Pottawatamie). Frontier Guardian. Semi-monthly. Whig. Circulation 3,000 .

Davenport (Scott). Davenport Gazette. Weekly. Whig. Circulation 600.

Davenport (Scott). Democratic Banner. Weekly. Democrat. Circulation 300.

Keosaugua (Van Buren), Keosaugua Jeffersonian. Weekly. Democrat. Circulation 500.

(Wapello County) [Ottumwa]. Des Moines Republic. Weekly. Democrat. Circulation 816 .

(Wapello County) [Ottumwa]. Des Moines Courier. Weekly: Whig. Circulation $\mathbf{7 5 0}$.

LAY \& Brother, compilers. The Newspaper Record, containing a complete list of Newspapers and Periodicals in the United States, Canada and Great Britain. ... Philadelphia, 1856. (The Iowa section is on pages 14-15.)

Albia (M o n roe). Independent Press.

Bloomfield (Davis). True Flag.

Bluff City (Potawatamie). Chronotype.

Bluff City (Potawatamie). Bugle.

Burlington (Des Moines). Hawkeye.

Burlington (Des Moines). State Gazette.

Burlington (Des Moines). Telegraph.

Burlington (Des Moines). Farmer \& Horticultural List.

Camanche (Clinton). Camanche Chief.

Cedar Rapids (Lima). [Sic.] Cedar Valley Times.

Cedar Rapids (Lima). [Sic.] Era.

Council Bluffs (Potawatamie). Bugle.

Davenport (Scott). Gazette.

Davenport (Scott). State Democrat.

Davenport (Scott). Courier.
Davenport (Scott). Temperance Organ.

Davenport (Scott). Der Demokrat (Ger.).

Davenport (Scott). Banner.

Delphi (Delaware). Argus.

Delphi (Delaware). Republican.

Dubuque (Dubuque). Tribune.

Dubuque (Dubuque). Herald.

Dubuque (Dubuque). Express.

Dubuque (Dubuque). Observer.

Eddyville (Wapello). Free Press.

Elkader (Clayton). Tribune.

Fairfield (Jefferson). Ledger.

Fairfield (Jefferson). Prairie Farmer.

Fairfield (Jefferson). Sentinel.

Fort Des Moines (Polk). Statesman.

Fort Madison. (Lee). Christian Evangelist.

Fort Madison (Lee). Plain Dealer.

Fort Madison (Lee). Times.

Fort Madison (Lee). Argus.

Garnaville (Clayton). Herald. 
Independence (Buchanan). Civilian.

Indianola (Warren). Republican. :

Indianola (Warren). Reporter.

Indianola (Warren). Journal.

Keokuk (Lee). Times.

Keokuk (Lee). Gate City.

Keokuk (Lee). Des Moines Valley Whig.

Keokuk (Lee). Dispatch.

Keosauque (Van Buren). Republican.

Knoxville (Marion). Journal.

Lancaster (Keokuk). Friend.

Lansing (Allomakee). Intelligeneer.

Marion (Linn). Register.

Mt. Pleasant (Henry), Observer.
Mt. Pleasant (Henry). True Democrat.

Muscatine (Muscatine). Enquirer. Muscatine (Muscatine). Journal. Newton (Jasper). Express.

Oskaloosa (Mahaska). Horald.

Oskaloosa (Mahaska). Times.

Ottumwa (Wapello). Courier.

Pella (Marion). Gazette.

Preparation (Monona). News \& Messenger.

St. Marys (Mills). Gazette.

Sidney (Fremont). Journal.

Tipton (Cedar). Advertiser.

Vernon (Van Buren). Democratic Mirror.

Vinton (Benton). Eagle.

Wapello (Louisa). Intelligencer.

Washington (Washington). Argus.

Kenny, Daniel J., compiler. The American Newspaper Directory and Record of the Press, containing an accurate list of all the Newspapers, Magazines, Reviews, Periodicals, etc., in the United States \& British Provinces of North America. . . New York: Watson \& Co., Publishers, 102 Nassan Street, 1861. ('The Iowa section is on pages 21-23.)

Afton. Eagle. Weekly.

Albia (Mon roe). Republican. Weekly.

Anamosa Court House. Gazette. Weekly.

Anamosa Court House. Eureka. Weekly.

Bedford. South Western. Weekly.

Bellevue. Journal. Weekly.

Bloomfield. Clarion. Weekly.

Bloomfield. World's Ozon. Weekly.

Boonsboro [Boone Co.]. News. Weekly.

Bradford [Chickasaw Co.] Nezes. Weekly.

Burris [Louisa Co.], Iowan. Weekly.
Burris [I.ouisa Co.]. Reporter. Weekly.

Burlington. Gazette. Daily.

Burlington. Hackeye. Daily and weekly.

Burlington. Telegraph. Weckly.

Camanche (Clinton). Chief. Weekly.

C a m a n c h e (Clinton). Citizen. Weekly.

Camanche (Clinton), Democrat. Weekly.

Cedar Falls (Black Hawk). Banner. Weekly.

Cedar Falls (Black Hawk). Democrat. Weekly.

Cedar Rapids (Linn). Banner. Weekly. 
Cedar Rapids (Linn). Gazette. Dubuque. Farmer. Weekly. Weekly.

Cedar R apids (Limn). Times. Weekly.

Centerville. Chieftain. Weekly.

Centerville. Remublican. Weekly.

Chariton Court House. Patriot. Weekly.

Dubuque. Herald. Daily and weekly.

Dubuque. Times. Daily and weekIy.

Dubuque. States-Zeitung [German]. Weekly.

Dubuque. Tribune. Weekly.

Dyersville. Mercury. Weekly.

Eddyville. Commercial. Weekly.

Fldora. Sentinel. Weekly.

Elkader. Tribune. Weekly.

Fairfield. Jeffersonian. Weekly.

Fairfield. Ledger. Weekly.

Fayette. Journal. Weekly.

Fayette. Observer. Weekly.

Fort Dodge. Republican. Weckly.

Fort Dodge. Sentinel. Weckly.

Fort Madison. Evangelist. [Religious.] Monthly.

Fort Madison. Plaindealer. Weekly.

Guttenburgh. Journal. Weekly.

Guttenburgh. Register. Weekly.

Hamilton [Webster City]. Freeman. Weekly.

Hampton. Rocord. Weekly.

Hardin. Courier. Weekly.

Independence. American Eagle. Weekly.

Independence. Civilian. Weekly.

Indepondence. Guardirn. Weekly.

Independence. The Rising Tide. Weekly.

Indianola Court House. Visitor. Weekly.

Iowa City, Reporter. Weekly.

Iowa City. Republican. Weekly.

Iowa City. State Press. Tri-weekly.

Keosauqua Court House, Neros. Weekly.

Keosauqua Court House. Republican. Weekly.

Keokuk. Gate City. Daily and weekly.

Dubuque. Christian Witness. Weekly.

Des Moines. Statesman. Daily.

Des Moines. iRegister. Weekly.

Dewitt. Journal. Weekly.

Dewitt. Standard. Weekly. 
Keokuk. Journal. Daily and weekly.

Keokuk. Post. Weekly.

Keokuk. Whig Valley. Weekly.

Knoxville. Journal. Weekly.

Knoxville. Republican. Weekly.

Lafayette [now Albion, Marshall Co.]. Journal. Weekly.

Lansing (Allamakce). Mirror. Weekly.

Le Claire (Scott). Express. Weekly.

Le Claire (Scott). Republican. Weekly.

Lyon [Leon] (Decatur). Pioneer. Weekly.

Lyons [Clinton Co.]. Advocate. Weekly.

Lyons [Clinton Co.]. Mirror. Weekly.

Magnolia. Republican. Weekly.

Marengo. Visitor. Weekly.

Marion. Herald. Weekly.

Marion. Register. Weckly.

Marietta [Marshall Co.] Express. Weekly.

Marshalltown. Times. Weekly.

Maquoketa. Excelsior. Weekly.

Maquoketa. Sentinel. Weekly.

Milwaukie. ${ }^{1}$ Irrepressible Conflict. Weekly.

Mitchcll. Republican. Weekly.

Montezuma. Gazette. Weekly.

Montezuma. Remeblican. Weekly.

Muscatine. Enquirer. Wcekly.

Muscatine. Journal. Weckly.

MeGregor. Press. Weekly.

MeGregor. Times. Weckly.

Mt. Ayr. Republican. Weekly.

Mt. Pleasant. Iotoa Farmer. Weekly.

Mt. Pleasant. Home Journal. Weekly.
Mt. Pleasant. Tribune. Weckly.

M't. Vernon. News. Weekly.

Nevada City. Advocate. Weekly.

Newton. Free Press. Weekly.

New Hampton. Courier. Weekly.

New Oregon [Howard Co.]. Plain Dealer. Weekly.

Osage. Iorean. Weekly.

Osceolat Conrier. Weekly.

Oskaloosa. Herald. Weekly.

Oskaloosa. Times. Weekly.

Onawee [Onawa]. The Monona Cordon. Weekly.

Ottumwa. Courier. Weekly.

Ottumwa. Statesman. Weekly.

Pacific [Mills Co.]. Herald. WeekIy.

Pella (Marion). Gazette. Weekly.

Sabula (Jackson). Tribune. Weekly.

Sidney. Herald. Weekly.

Sidney. Journal. Weekly.

Sigourney. Democrat. Weekly.

Sigourney. Life in the West: Weekly.

Sigourney. Neros. Weekly.

Sioux City. Eagle. Weekly.

Sioux City. Register. Weekly.

St. Charles [Charles City]. Intolligencer. Weekly.

Tipton. Advertiser. Weekly.

'Tipton. Democrat. Weekly.

Toledo. Transcript. Weekly.

Vernon Springs [Howard Co.].

Eaglo. Weekly.

Vinton. Democrat \& Eaglo. Weekly.

Wapello. Gazette \& Ropublican. Weekly.

Washington. Herald \& Press. Weekly.

Waterloo. Courier. Weekly.

Waterloo. Register. Weekly.

1The locations of nearly all of the newspapers in these lists are identified either by the name of a well known city or town now in existence, or by the name of the county. A few were located at towns long since abandoned and now unknown to most reaclers. Where we know the location of such places we have supplied the name of the county in brackets. Where we have not known the location, as this one, we say so in a footnote--EdTor Annals. 


\section{DIRECTORIES OF EARLY IOWA NEWSPAPERS}

Waverly. Intelligencer. Weekly. Waverly. Republican. Weekly. Webster City. Courier. Weekly. Webster City. Freeman. Weekly. West Union. Courier. Weekly.
West Union. Freeman. Weekly. West Union. Public Reviero. Weekly.

Winterset. Madisonian. Weekly.

Geo. P. Rowell \& Co., compiler. American Newspaper Directory, containing accurate lists of all the newspapers and periodicals published in the United States and Territories. ... New York: Geo. P. Rowell \& Co., . . 1869. (Iowa section on pages $31-36$.)

The preface of this volume is dated January 1, 1869, so the listings apparently indicate the status of the papers at the end of 1868. This directory was checked most. conscientiously and is based on information received directly from the publishers of the papers listed. For each paper there is given the name, frequency, politics, number of pages, page size, subscription price, year of establishment, personnel of editorial and publishing auspices, and the amount of circulation claimed. There are given here only the data which seem germane to the present purpose: the place of publication, the name of the paper, the frequency, politics, the year of establishment, and the personnel. The other information can always be obtained by interested investigators from the Directory itself.

Adel. Dallas Qcaette. Weekly on Fridays. Republican. Established 1866. G. A. Atwood, editor and publisher.

Afton. Reveille. No report.2

Afton. Tribune. Weekly on Thursdays. Republican. Established 1868. W. R. Roberts, editor and publisher.

Albia. Republic. Weekly on Thursdays. Democrat. Established 1868. A. C. Bailey, editor and publisher.

Albia. Union. Weekly on Thursdays. Republican. Established 1860. Val. Mendel, editor and publisher.

Algona. Upper Des Moines. Weekly on Wcdnesdays. Republican. Established 1865. James H. Warren, editor and publisher.

Anamosa. Eureka. Weekly on Thursdays. Republican. Established 1856. E. Booth \& Son, editors and publishers.

Barclay [Black Hawk Co.]. Star of the Evening. No report.

Bedford. Iotea South-West. Weekly on Saturdays. Established 1867. Lucas \& Patrick, editors and publishers.

Bedford. National Express. No report.

2The words "no report" mean that such a paper was known to have been published but that the editor could not confirm the fact that it was still being published at the time the Directory went to press. 
Belle Plaine, Benton County Transcript. No report.

Bellevuc. Jonrnal. Weekly on 'Thursdays. Republican. Established 1862. W. \& E. Pollock, editors and publishers.

Bellevue. Union Guard. No report.

Bentonsport. Sigmal. No report.

Bloomfield. Davis Comnty Republican. Weekly on Thursdays. Republican. Established 1863. E. 1. White, editor and publisher.

Boonesborough. Bome County Advocate. Weekly on 'Thursdays. Rcpublican. Established 1865. B. F. Hilton, editor and publisher.

Brighton. Pioneer. Weekly on Fridays. Independent. Established 1868. R. H. Moore, editor and publisher.

Burlington. Gazelte and Argus. Daily except Monday. Weekly on Thursdays. Democrat. Established 1838. Barrett, Barker \& Co., editors and publishers.

Burlington. Hazck-Eye. Daily except Monday. Semi-weekly on Wednesdays and Saturdays. Weekly on Thursdays. Republican. Established 1839. Edwards \& Beardsley, editors and publishers.

Burlington. Iorea Tribune. Tri-weekly on Tuesdays, Tlursdays and Saturdays. Weekly on Wednesdays. German. Republican. Established 1854. DeWolf \& Sington, editors and publishers.

Carroll. Western Hermilt. Weekly on Wednesdlays. I. F. H. Sugg, editor and publisher.

Cedar Falls. Gazette. Weekly on Fridays. Republican. Established 1859. C. W. \& E. A. Snyder, editors and publishers.

Cedar Rapids. Times. Weekly on Thursclays. Republican. Established 1850. Dr. F. McClelland, editor. Ayers Bros., publishers.

Cedar Rapids. Westem World. Weekly on Saturdays. Republican. Established 1868. Ballard \& Enos, editors and publishers.

Centreville. Loyal Citizen. Weekly on 'Tuesdays. Republican. Established 1864. M. M. Waldon, editor and publisher.

Chariton. Democrat. Weekly on Saturdays. Democrat. Established 1867. J. V. Faith, editor and publisher.

Chariton. Patriot. Weekly on Wednesdays. Republican. Established 1858. Ragsdale Bros., editors and publishers.

Charles City. Advocate. No report.

Charles City. Intelligencer. Weekly on 'Thursdays. Republican. Established 1856. A. B. F. Hildreth, editor and publisher.

Charles City. Post. No report.

Ciarinda. Page Connty Democrat. Weekly on Thursdays. Democrat. Established 1868. Jas. Arrick and H. Houston, editors and publishers.

Clarkesville. Star of the West. Weekly on Fridays. Republican. Established 1868. Frank Case, editor and publisher.

Clermont. Fayette County Leader. No report.

Clinton. Catholic Roviow. Weekly on Saturdays. Catholic. Established 18(i8. James Glynn, editor and publisher.

Clinton, Herald. No report. 
Clinton. Iowa Age. Weekly on Fridays. Democrat. Established 1868. E. H. Thayer, editor and publisher.

Clinton. Iozea Volks Zeitung. Weckly on Thursdays. German. Established 1867. F. G. Pffeiffer, editor and publisher.

Corydon. Monitor. Weekly on Saturdays. Republican. Established 1863. L. Mills, Jr., and Mary Hartshorn, editors and publishers.

Council Bluffs. Bugle. Daily and weekly on Thursdays. Democrat. Established 1849. L. W. Babbitt, editor; C. H. Babbitt, publisher.

Council Bluffs. Nonpareil. Daily except Monday. Tri-weekly on Tucsdays, Thursdays and Saturdays. Weekly on Saturdays. Established 1856. J. W. Chajman, editor-in-chief; Chapman, Gray \& Mill, publishers.

Cresco. Iozea Plain Dealer. Weekly. Democrat. Established 1859. Mead \& Brown, editors and publishers.

Cresco. Times. Weekly on Thursdays. Republican. Established 1867. J. A. Hoxie, editor and publisher.

Dakota City. Humboldt County Independent. Weekly on Fridays. Republican. Established 1868. M. D. Williams, editor; Chas. Bergk, publisher.

Davenport. Democrat. Daily except Sunday. Weckly on 'Thursdays. Democrat. Established-daily 1855, weekly 1838. Richardson Bros., editors and publishers.

Davenport. Der Demokrat. Daily except Monday. Weekly on Thursdays. German. Republican. Established 1851. J. B. Stibolt, editor; H. Lischer, publisher.

Davenport. Home Visitor. No report.

Davenport. Gazette. Daily except Sunday. Weekly on Wednesdays. Republican. Established-daily 1854, weekly 1841. Edward Russell, editor; Gazette Co., publishers.

Davenport. True Radical. Weckly on Saturdays. Established 1867. Frank L. Jervis, editor; Jervis \& Smallfield, publishers.

Davenport. Western Soldiers' Friend and Fireside Companion. Weekly on Saturdays. Republican. Established 1867. C. A. Haviland, editor; Haviland \& Co., publishers.

Decorah. Democrat. Weekly on 'Tuesdays. Democrat. Established 1868. Robert Shurley, editor and publisher.

Decorah. Register. Weekly on Tuesdays. Republican. Established 1866. Geo. W. Haislet, editor and publisher.

Decorah. Republican. Weckly on Fridays. Republican. Established 1859. A. K. Bailey \& Bros., editors and publishers.

Decorah. Ved Armen. Norwegian. B. Anundson, editor and publisher.

Decorah. Kirkelig Maanedstidende. Norwegian. Semi-monthly. I.utheran. Established 1855. Norwegian Lutheran College Ficulty, editors. Rev. N. Brandt, publisher.

Denison. Revieze. Weekly on Fridays. Established 1867. G. N. Stephens, editor; Money \& Stephens, publishers. 
Des Moines. Iotca Statesman. Daily except Sunday. Weekly on Thursdays. Democrat. Established 1835. Staub \& Jenkins, editors and publishers.

Des Moines. Iozo Slate Register. Ditly except Monday. Weekly on Tuesdays. Republican. Established 1856. Mills \& Co., editors and publishers.

Des Moines. Lowa Homestead. Weekly on Fridays. Agricultural. Established 1855. Dr. G. Sprague \& Co., editors and publishers.

Des Moines. Iowa Temperance Standard. Weekly on Saturdays. 'lemperance. Established 1865. Wm. H. Fleming and J. M. Dixon, editors. Grand Lodge of Iowa, I. O. G. T., publishers.

Des Moines. Iowa Instructor. Monthly. Mills \& Co., publishers. Organ of the superintendent of public instruction.

Des Moines. National Platform. Monthly. Established 1867. Platform Publishing Co., editors and publishers. Devoted to temperance and masonic.

Des Moines. Western Jurist. No report.

De Witt. Obsorver. Weekly on Wednesdays. Republican. Established 1864. S. H. Shoemaker, editor and publisher.

Dubuque. Herald. Daily. Weekly on Wednesdays. Democrat. Establislıed 1851. M. M. Ham, editor; Ham \& Carver, publishers.

Dubuque. Times. Dialy except Monday. Weekly on Wednesdays. Republican. Established 1857. M. S. Barnes, editor; Barnes \& Ryan, publishers.

Dubuque. Jozea Stauls Zeitang. Weekly on Saturdays. German. Republican. Established 1863. Wm. Meher, editor and publisher.

Dubuque. National Demokrat. Weekly on 'Thursdays. German. Democrat. Established 1856. F. A. Gniffke, editor and publisher.

Eddyville. Des Monines Valley Gazoto. Weekly on Fridays. Republican. Established 3868. J. W. Stright, editor and publisher.

Eddyville. Transcript. Weekly on 'Thursdays. Republican. Established 1867. A. C. Jones, editor and publisher.

Eldora. Leader. No report.

Elkader. Clayton Comnty Journal. Weekly on Wednesdays. Republican. Established 1853. Joseph Eiboeck, editor and publisher.

Elkader. Der Nord Lozoa Herald. Weekly on Fridays. German. Republican. Established 1868. Papin \& Quinte, editors and publishers.

Estherville. Northern Vindicator. Weekly on Wednesdays. Republican. Established 1868. Northrop \& Bates, editors and publishers.

Fairfield. Iozea Domocrat. Weekly on Saturdays. Democrat. Established 1866. N. N. Bleakmore, editor and publisher.

Fairfield. Jefferson Connty Home Visitor. No report.

Fairfield. Ledlger. Weekly on Thursclays. Republican. Established 1851. W. W. Junkin, editor and publisher.

Fayette. North Iozea Observer. Weekly on Thursdays. Established 1867. Wood \& Cole, editors and publishers.

Fontanelle. Adair County Register. Weekly on 'Thursdays. Republican. Established 1863. Kilburn \& Rutt, editors and publishers. 
Forest City. Winnebago Press. Weekly on 'lhursdays. Republican. Established 1867. Arthur Linn, editor; Linn \& Hawood, publishers. Fort Dodge. Jozea North-lWest. Weekly on Wednesdays. Republican Established 186i3. 13. F. Gue, editor and publisher.

Fort Madison. Lozo State Bulletin. No report.

Fort Madison. Plain Dealor. Weekly on 'Thursdays. Republican. Established 1852. J. C $\mathrm{r}$. Willson, editor and publisher.

Glenwood. Opinion. Weekly on Saturdays. Republican. Established 1864. Morgan \& I.unt, editors and publishers.

Grundy Center. Ginudy Counly Allas. Weekly on Fridays. Established 1868. L. D. 'I'racy, editor; 'Tracy \& Peckham, publishers.

Hamburg. Fremont Weekly Times. No report.

Hampton. Franktin Roporter. Weekly on 'I'hursdays. Republican. Established 1866. J. C. Whitney, editor and publisher.

Independence. Buchanan Connty Bulletin. Weekly on Fridays. Repuhlican. Established 1865. J. L. Loomis, editor and publisher.

Independence. Consorvative. Weckly on Wednesdays. Democrat. Established 1854. Barnhart Bros. \& Co., editors and publishers.

Indianola. Bamuer. No report.

Iowa City. Republican. Weckly on Wednesdatys. Established 1849. $\mathrm{N}$. H. Brainerd, editor and publisher.

lowa City. Slate Press. Weekly on Wednesdays. Democrat. Istablished 1860. John P. Irish, editor and publisher.

lowa City. Annals of Iowa. Quarterly. Established 1863. Sanford W. Huff, M. D., editor; State Historical Society, publishers.

lowa Falls. Sentinel. Weekly on Wednesdays. Republican. Established 1855. M. C. Woodruff, editor and publisher.

Jefferson. Rra. Weekly on Wednesdays. Republican. Established 1860. M. I-I. \& M. I. Money, editors and publishers.

Keokuk. Constitution. Daily except Monday. Weekly on Wednesdays. Democrat. Fstablished 1852. Thos. W. Colgett and Chas. Smith, editors and publishers.

Keokuk. Gate City. Daily except Monday. Weekly on Wednesdays. Republican. Established 1846. J. B. Howell, editor and publisher; S. M. Clarke, associate editor.

Keokuk. Tolegraph. Weekly on Saturdays. German. Tstablished 1865. Jacob .J. Wohlmend, editor and publisher.

Keosauqua. Republican. Weekly on 'I'hursdays. Republican. Rstablished 1855. .T. Mayne, editor and publisher.

Knoxville. Iozea Voter. Weckly on Thursdays. Republican. IEstablished 1867. Sperry \& Barker, editors and publishers.

Knoxville. Marion County Democrat. Weekly on 'Tuesdays. Democrat. Established 1864. T. I.. MeCormack, editor and publisher.

Lansing. Chronicle. Weekly on 'l'hursdays. Independent. Established 1858. Haislet \& Taylor, editors and publishers.

Lansing. Mirror. Weekly on Tuesdays. Republican. Established 1855. T. C. Medary, editor and publisher. 
Lansing. Union. No report.

LeClaire. Register. Weekly on Saturdays. Established 1866. J. Gamble, editor; Register Printing Co., publishers.

Leon. Decalur County Journal. Weekly on 'Ihursdays. Republican. Established 1868. P. O. James, editor and publisher.

L.eon. Pioneor. Weekly on 'l'uesdays. Established 1865. G. N. Udell, editor and publisher.

Lewis. Cass County Messenger. Weekly on Saturdays. Republican. Established 1865. H. C. Johnson, editor and publisher.

Lynnville. Gazette. Weekly on Thursdays. Established 1868. Evans \& A rnold, editors and publishers.

Lyons City. Advocate. Weekly on Wednesdays. Democrat. Established 1866. A. P. Durlin, editor and publisher.

Lyons City. Bamer. No report.

Lyons. Weekly Mirror. Weekly on Saturdays. Republican. Established 1854. Beers \& Eaton, editors and publishers.

Magnolia. Harrison County Union. No report.

Magnolia. Western Star. Weekly on Saturdays. Republican. Established 1866. Cook \& Waterman, editors and publishers.

Manchester. Delaware County Union. Weekly on Thursdays. Republican. Established 1864. L. L.. Ayers, editor and publisher.

Maquoketa. Excelsior. Weekly on 'Thursdays. Republican. Established 1855. Wm. H. McCarron, editor and publisher.

Maquoketa. Jackson Connty Sentinel. No report.

Marengo. Cilizen. Weekly on Fridays. Democrat. Established 1866. E. C. Upham, editor.

Marengo. Lowa Valley Review. No report.

Marengo. Progressize Republican. Weekly on Wednesdays. Republican. Established 1862. Spering \& Crenshaw, editors and publishers.

Marion. Limn County Patriot. No report.

Marion. Register. Weekly on Wednesdays. Republican. Established 1852. S. W. Rathburn, editor and publisher.

Marion. Hope of Israel. Semi-monthly. Seventh day advent. Established 1866. B. F. Snook, editor; A. Aldrich, publisher.

Marshalltown. Marshall County Advance. Weekly on Tuesdiys. Democrat. Established 1866. Barnhart Bros. \& Carleton, editors and publishers.

Marshalltown. Marshall County Times. Weekly on Saturdays. Republican. Established 1858. Chas. Aldrich, editor and publisher.

Mason City. Cerro Cordo Republican. Weekly on Thursdays. Republican. Established 1861. Noyes \& Sirrine, editors and publishers.

McGregor. Commercial. No report.

MeGregor. Neres. Weekly on Saturdays. Republican. Established 1861. R. Hamilton, editor; McGregor News Printing Co., publishers.

McGregor. North Iowa Times. Weekly on Wednesdays. Democrat. Established 1856. A. P. Richardson and J. H. Andrick, editors and publishers. 
Mechanicsville. Journal. Weekly on Thursdays. Republican. Established 186\%. Andrews \& Golding, editors and publishers.

Montana [Boone]. Standard. Weekly on Saturdays. Republican. Established 1865. W. H. Gallup, editor and publisher.

Montezuma. Republican. Weekly on Wednesdays. Republican. Established 1856. Grove \& Dalbcy, editors and publishers.

Monticello. Express. Weekly on 'Thursdays. Democrat. Established 1864. G. W. Hunt, editor and publisher.

Mt. Ayr. Republican. No report.

Mt. Ayr. Ringgold Record. Weekly on 'Thursdays. Established 1865. Geo. 13. Roby, editor and publisher.

Nit. Pleasant. Henry County Press. Weekly on Wednesdays. Republican. Established 1866. O. H. Snyder, editor; Snyder Bros, publishers.

Mt. Pleasant. Home Journal. No report.

Muscatine. Courior. Daily except Monday. Weekly on 'T'hursdays. Democrat. Established 1861. Barnhart Bros. \& Witmer, editors and publishers.

Muscatine. Journal. Daily except Sunday. 'Tri-weekly on 'Tuesdays, Thursdays and Saturdays. Weekly on Fridays. Republican. Established 1851. Mahin Bros., publishers.

Nashua, Post. Weekly on Fridays. Republican. Established 1867. A. Felt, editor and publisher.

Nevidia. Story County Aegis. Weckly on Wednesdays. Republican. Eistablished 1856. V. A. Ballou, editor and publisher.

New Hampton. Courior. Weekly on Fridays. Republican. Estahlished 1861. Geo. M. Reynolds, editor and publisher.

Newton. Jasper Free Press. Weekly on Thursdays. Republican. Wstablished 1859. W. S. Benham, editor and publisher.

Newton. Jasper Republican. Weekly on Fridays. Republican. Established 1867. Besack, Allum \& Rodgers, editors and publishers.

Onawa. Monona Connty Gezette. Weekly on Thursdays. Republican. Established 1864. W. A. Greene, editor; Aldridge \& Greene, publishers.

Orford. Leuder. Weekly on Tuesdays. Republican. Established 1868. Wm. M. Patrick, editor and publisher.

Osage. North Tozoan. Weekly on Fridays. Republican. Established 1865. W. Toman, editor and publisher.

Osceola. Clarke County Sentimel. Weekly on Fridays. Republican. IEstablished 1863. Dague \& 'Thompson, editors and publishers.

Oskaloosa. Central Ioza Citizen. Weekly on Saturdays. Republican. Established 1867. M. G. Carleton, editor. Carleton \& Bacon, publishers.

-Oskaloosa. Democratic Conservator. Weekly on Wednesdays. Democrat. Established 1866. P. C. Welch, editor and publisher.

Oskaloosa. Herald. Weekly on Thursdays. Republican. Established 1850. Hunter, Leighton \& Needham, editors and publishers. 
Oskaloosa. Evangelist. Monthly. Church of Christ. Established 1865. Allen Hickey, editor and publisher.

Oskaloosa. Temperance Gem. No report.

Oskaloosa. Watchman. No report.

Ottumwa. Courier. Daily except Sunday. Weekly on Thursdays. Republican. Established 1847. John M. Hedrick, editor. Caldwell, Holden \& Co., publishers.

Ottumwa. Copper Head. Weekly on Wednesdays. Democrat. Established 1868. M'Cully \& Evans, editors and publishers.

Panora. Guthrie County Ledger. No report.

Panora. Guthrie County Nezes. No report.

Panora. Guthrie Vedette. Weekly on Thursdays. Republican. Established 1865. Lew. Apple, editor and publisher.

Parkersburg. Quide. Weekly on Saturdays. Republican. Established 1868. Wm. W. Yarham, editor and publisher.

Pella. Blade. Weekly on Wednesdays. Republican. Established 1865. Curtis \& Betzer, editors and publishers.

Pella. Gazette. Weekly on Thursdays. Hollandish. Republican. Established 1866. N. J. Gesman, editor; G. van Ginkel, publisher.

Pella. Le Glaneur Missionaire. No report.

Pella. Weehblad. Weekly on Tuesdays. Hollandish. Democrat. Established 1861. Henry Hospers, editor and publisher.

Quincy. Adams County Gazette. Weekly on Thursdays. Republican. Established 1867. Webster Eaton, editor and publisher.

Red Oak Junction. Montgomery County Express. Weekly. Republican. Established 1868. Webster Eaton, editor and publisher. (Printed at the office of the Quincy Qazette.)

Sabula. Eastern Iozan. Weekly on Saturdays. Independent. Established 1869. J. F. H. Sugg, editor and publisher.

Sabula. Gazetle. Weekly on Saturdays. Established 1866. J. E. Kolp $\&$ Co., editors and publishers.

Sand City." Union. No report.

Sand Spring. ${ }^{4}$ Sentinel. No report.

Sidney. American Union. Weekly on Thursdays. Democrat. Established 1864. J. A. Bodenhamer, editor; D. \& G. Bodenhamer, publishers.

Sigourney. Keokuk County Neres. No report.

Sioux City. Journal. Weekly on Thursdays. Republican. Established 1864. Mahlon Gore, editor and publisher.

Sioux City. Register. Weekly on Saturdays. Democrat. Established 1858; F. M. Ziebach, editor; Wm. Freney, publisher.

Springvale. Humboldt County True Democrat. Weekly on Fridays. Republican. Established 1866. S. H. Tuft, editor and publisher.

St. Charles. Madison County Journal. No report.

Steamboat Rock. American Citizen. Weekly on Wednesdays. Democrat. Established 1868. Deloss S. Ring, editor and publisher.

3Location not known.

4Location not known. Possibly Lime Spring, Howard County. 
Tama City. Tama County Union. Weekly on Thursdays. Republican. Established 1866. Cyrus B. Ingham, editor and publisher.

'Tipton. Advertiser. Weekly on Thursdays. Republican. Established 1854. Clark \& Mulford, editors and publishers.

Toledo. Tuma County Republican. Weekly on Thursdays. Republican. Established 1867. M. B. C. True, editor and proprietor.

Vinton. Benton County Nezss. No report.

Vinton. Eagle. Weekly. Republican. Established 1855. David H. Frost, editor; Hanford \& Frost, publishers.

Wapello. Republican. Weekly on Saturdays. Established 1857. L. W. Myers, editor and publisher.

Washington. Record. Weekly on Saturdays. Republican. Established 1867. A. S. \& S. P. Bailey, editors and publishers.

Washington. Washington County Press. Weekly on Wednesclays. Republican. Estabiished 1855. H. A. Burrell, editor and publisher.

Waterloo. Courier. Weekly on Thursdays. Republican. Established 1858. J. W. Logan, editor; Hartman \& Logan, publishers.

Waukon. Standard. Weekly on Thursdays. Republican. Established 1868. R. L. Hayward and A. M. May, editors and publishers.

Waverly. Democratio Neros. Weekly on Thursdays. Democrat. Established 1867. Geo. Lindley, editor and publisher.

Waverly. Republican. Weekly on Thursdays. Republican. Established 1855. E. C. Moulton, editor; Stewart \& Moulton, publishers.

Webster City. Hamilton Freeman. Weekly on Wednesdays. Republican. Established 1857. John D. Hunter, editor and publisher.

West Mitchell. Mitchell Connty Press. Weekly on Thursdays. Republican. Established 1865. F. M. Atherton, editor and publisher.

West Liberty. Mnscatine County Enterprise. No report.

West Union. Fayette County Umion. Weekly on Wednesdays. Democrat. Established 1865. McClintock Rickel \& Wood, editors and publishers.

West Union. Republican Gazetie. Weekly on Saturdays. Republican. Established 1867. Shannon \& Tulmadge, editors and publishers.

West Union. Iotva Public Record. No report.

Wheatland. Clinton Advocale. No report.

Wilton. Chronicle. Weekly on Thursdays. Republican. Established 1867. Eaton \& Baker, editors and publishers.

Winterset. Hazk-Eye Flag. No report.

Winterset. Madisoniam. Weekly on Thursdays. Established 1856. Davies \& Ewing, editors and publishers. 
Copyright of Annals of Iowa is the property of State of Iowa, by \& through the State Historical Society of Iowa and its content may not be copied or emailed to multiple sites or posted to a listserv without the copyright holder's express written permission. However, users may print, download, or email articles for individual use. 